ORIGINAL ARTICLE

\title{
Expression and Location of Glucose-regulated Protein 78 in Testis and Epididymis
}

\author{
W Wang ${ }^{1 *}$, X Wang ${ }^{2 *}$, P Zhu ${ }^{1}, \mathrm{CM} \mathrm{Sun}^{3}, \mathrm{~S} \mathrm{Jin}{ }^{1}, \mathrm{~J} \mathrm{Liu}^{1}, \mathrm{~N} \mathrm{Li}^{1}, \mathrm{~J} \mathrm{Liu}^{3}$
}

\begin{abstract}
Objective: To know the role of glucose-regulated protein 78 (GRP78/BiP/HSPA5) in spermatogenesis and its expression and location in the testis and epididymis.

Methods: Immunohistochemistry and Western blot were used to detect GRP78 location and expression in the testis and epididymis.

Results: Glucose-regulated protein 78 was observed in spermatocytes, round spermatids and interstitial cells of the testis and in principal cells of the epididymis. Glucose-regulated protein 78 was first detected in the rat testis at postnatal day 14. Thereafter, the protein level increased gradually with age and was maintained at a high and stable state after postnatal day 28. In the rat, GRP78 was expressed in the principal cells but not in clear cells of the epididymis.

Conclusion: Glucose-regulated protein 78 participates in the process of spermatogenesis.
\end{abstract}

Keywords: Epididymis, glucose-regulated protein 78 (GRP78), testis

WIMJ Open 2014; 1 (1): 14

\section{INTRODUCTION}

Mammalian heat shock proteins (HSPs) are evolutionarily highly conserved molecular chaperones (1). They are induced in cells exposed to elevations in temperature, chemical or physical stress, viral infection, drugs, transforming agents etc $(2,3)$. Physiological events such as cell growth, differentiation, development and ageing can also induce the synthesis of HSPs $(4,5)$.

A few investigations have shown that the $70 \mathrm{kDa}$ heat shock proteins (HSP70) are associated with cancer. For most cancer cells, the high expression of HSP70 is essential for their survival (6). Over-expression of HSP70 can increase the tumorigenicity of cancer cells (7). Recent studies have indicated the involvement of HSP70 family members during spermatogenesis and fertilization $(8,9)$. Heat shock protein 70 persists in ejaculated boar and bovine spermatozoa and is subjected to subcellular translocation during capacitation and acrosome reaction $(10,11)$. Moreover, the presence of a specific antibody against HSP70 lowers the fertilization rate

From: ${ }^{1}$ Yuhuangding Hospital Central Laboratory, Yantai, Shandong, China,

${ }^{2}$ Yuhuangding Hospital Reproduction Medical Centre, Yantai, Shandong, China and ${ }^{3}$ Yuhuangding Hospital Chip Laboratory, Yantai, Shandong, China.

Correspondence: Dr J Liu, Yuhuangding Hospital, Yantai Yuhuangding, No 20 East Road, Shandong Province, 264000, China. E-mail: liulocus@ 126.com

*Contributed equally to the manuscript. in vitro in the bull (9), which strongly supports its importance in fertilization events.

Glucose-regulated protein 78 (GRP78; also known as binding immunoglobulin protein (BiP) or HSPA5), as one of the more important members of the HSP70 family, is regarded as an essential housekeeping gene (12). Besides the correlation between induced expression of GRP78 and resistance to apoptotic death in somatic cells and tumours (13), the knockout of GRP78 induces the death of mouse embryos at embryonic day 3.5 (12) and GRP78 can also play a critical role during mouse spermatogenesis (8). In the human, GRP78 chaperone proteins express at the surface of oviductal epithelial cells and bind to spermatozoa $(14,15)$, which indicates that human GRP78 has a role in spermatogenesis and fertilization.

Mammalian spermatogenesis is a long and complex process involving mitotic expansion of stem cells, meiotic recombination, and the ensuing generation of numerous spermatozoa containing a haploid genome. Identification of proteins participating in spermatogenesis is essential to understand the complex molecular mechanisms controlling this process. To achieve this goal, we identified proteins by the technique of proteomic profile and an integrative bio-informatics methods; GRP78 was one of them. The aim of this study was to study the occurrence and origin of GRP78 protein and its function in the process of spermatogenesis. 


\section{SUBJECTS AND METHODS Biological material}

Human testicular and epididymal tissues were obtained from six fathers, aged 27 to 32 years, who had experienced accidental death and had no history of pathology that could affect reproductive functions. Male rats of 7, 14, 21, 28, 60 and 120 days were purchased from the Experimental Animal Centre of Binzhou Medical College, Yantai, People's Republic of China. All experiments were approved by the Ethics Committee of YuHuangDing Hospital.

\section{Immunohistochemistry of testis and epididymis}

Testicular and epididymal tissues were immersed in Bouin's fluid and embedded in paraffin by the hospital pathology department. Six-micrometre sections were then deparaffinized in toluene and rehydrated in alcohol. The 1:50 diluted anti-GRP78 antibody (sc-1501, Santa Cruz Biotechnology, California, USA) was applied overnight at $4{ }^{\circ} \mathrm{C}$. After three washes in PBS-T (phosphate-buffered saline (PBS) containing $0.05 \%(\mathrm{v} / \mathrm{v})$ Tween 20$)$, the sections were incubated for one hour at room temperature with peroxidase conjugated anti-goat IgG secondary antibody (ZhongShan Biotechnology Co, Beijing, China). The peroxidase activity was revealed by using a DAB kit (ZhongShan Biotechnology). Negative controls were performed by using the same concentration of commercial goat IgG instead of polyclonal antibodies of GRP78. Finally, sections were counterstained with Harris's haematoxylin and mounted in water-based medium containing glycerol and Mowiol (Calbiochem; EMD Biosciences Inc, La Jolla, California, USA) as preservatives. Slides were observed with a Leica microscope.

\section{Western blotting}

Samples containing $50 \mu \mathrm{g}$ protein from the 7-, 14-, 21-, 28-, 60- and 120-day rat testes and epididymides were electrophoresed on $15 \%$ SDS polyacrylamide gels and transferred to a polyvinylidene fluoride (PVDF) membrane filter (Millipore, Billerica, Massachusetts, USA). The filters were blocked in PBS containing 5\% (w/v) non-fat milk powder for one hour and then incubated for two hours with a 1:100diluted anti-GRP78 polyclonal antibody. They were washed three times with PBS. The filters were then incubated for one hour with horseradish peroxidase (HRP)-conjugated antigoat IgG (ZhongShan Biotechnology). Specific proteins were detected with a DAB kit. On the same gels, a 1:250diluted anti-GAPDH polyclonal antibody (sc-1615, Santa Cruz Biotechnology) was used as reference protein to verify the amount and integrity of the protein.

\section{RESULTS}

\section{The localization of GRP78 in the testis}

Glucose-regulated protein 78 was expressed in almost all cell types that colonized the seminiferous tubules with the exception of spermatogonia (Fig. 1B). A stronger GRP78 signal was observed in the cytoplasm of spermatocytes and

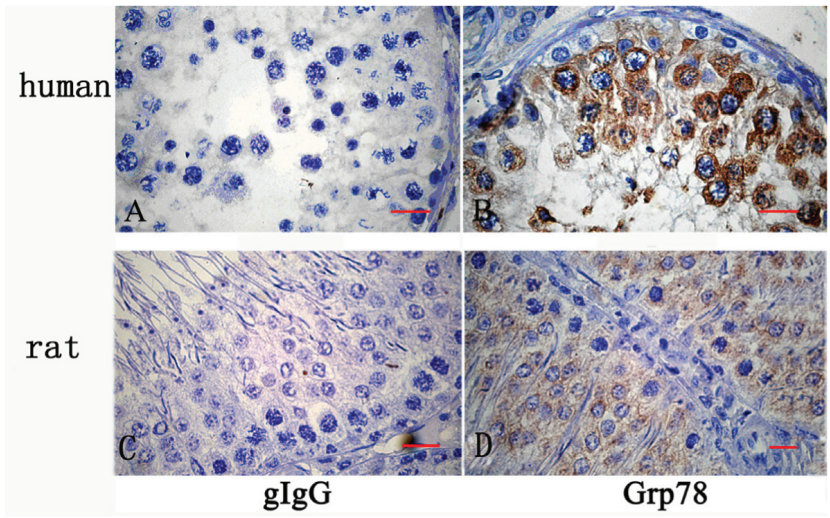

Fig. 1: Immunohistochemical localization of glucose-regulated protein 78 (GRP78) in the testis. Results were obtained using a polyclonal anti-GRP78 (B, D) and a goat IgG as a negative control (A, C). Positive staining is shown by a brownish precipitate. Bar represents $20 \mu \mathrm{m}$ (A and B).

round spermatids (Fig. 1D) forming a ring around the nucleus. As human testis of different ages was not available, the rat model was used to investigate the expression of GRP78 during testicular development. Western blot showed that GRP78 was first detected on postnatal day 14. Thereafter, the protein level increased gradually with age and maintained at a high and stable state after postnatal day 28 (Fig. 2A).

The result of immunohistochemistry of the testis was consistent with Western blot but revealed much more information. At day 28, GRP78 was detected in almost all the spermatogenic cells except the spermatogonia in the rat seminiferous tubules and located in the cytoplasm around the nucleus. A weak staining was also observed in the cytoplasm of interstitial cells (Fig. 2B).

\section{The localization of GRP78 in the epididymis}

Glucose-regulated protein 78 was expressed in epithelial cells of the caput, corpus and cauda epididymis and the signal increased gradually. A strong signal of GRP78 was detected in principal cells of the epididymis. In the caput, GRP78 was mainly detected in the cytoplasm in the infranuclear region whereas the signal was stronger in the cytoplasm in the supranuclear region in the corpus. In the cauda, the strongest GRP78 signal was observed on the apical side of the epithelial cells (Fig. 3).

It is noteworthy that in the caput epididymis of the rat, the lowest intensity was found in the caput while the highest was noted in the corpus and cauda segment. These results support the differential expression of GRP78 throughout the different anatomical segments of the epididymis. Western blot analyses were consistent with the results obtained by immunohistochemical approaches. In the clear cells of the rat corpus and cauda, there was a completely negative signal region with checkerboard pattern in the epithelium of the rat epididymis (Fig. 3). 
A

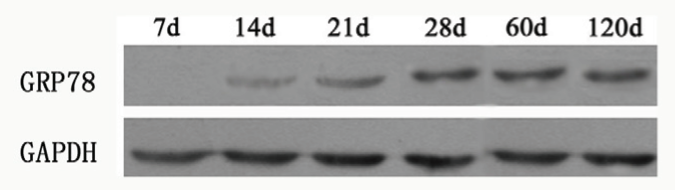

B

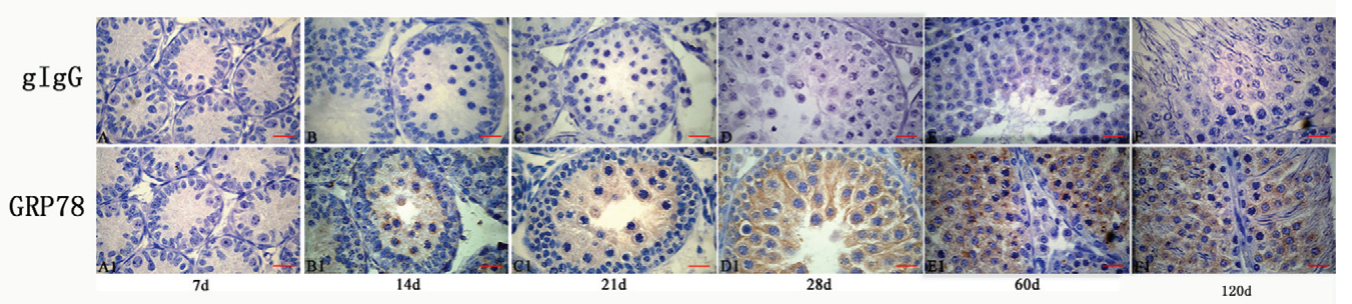

Fig. 2: (A) Western blot of glucose-regulated protein 78 (GRP78) protein expression in rat testes of different ages. The expression of GAPDH in corresponding tissues is displayed in the bottom panel as a control. (B) Immunohistochemical localization of GRP78 during the development of the rat testis. A1-F1: a polyclonal antiGRP78; A-F a goat IgG as a negative control. Positive staining is shown by a brownish precipitate. Bar represents $20 \mu \mathrm{m}$.

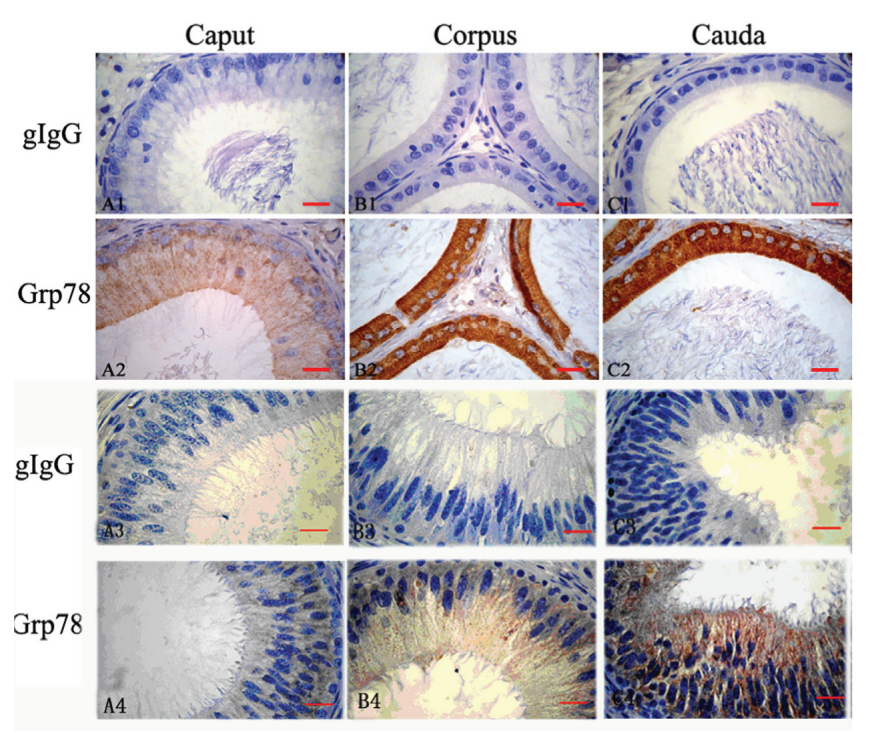

Fig. 3: Immunohistochemical localization of glucose-regulated protein 78 (GRP78) in the epididymis. The presence of GRP78 was assessed using a polyclonal anti-GRP78 (A2-C2; A4-C4) or goat IgG as a negative control (A1-C1; A3-C3). Brownish precipitate represents positive staining. Bar represents $20 \mu \mathrm{m}$.

\section{DISCUSSION}

Glucose-regulated protein 78 belongs to the HSP70 family. Members of this protein family are chaperones containing ATP binding/hydrolysis activities that mediate their ability to aid in protein folding, transport and assembly by coordinating the sequential binding and release of the protein substrate (16). In mice, the HSP70 family contains at least seven different proteins and several of them have been demonstrated to have a critical role in spermatogenesis. One example is HSP70-2 protein; its expression is high in pachytene spermatocytes throughout meiosis and remains in spermatids and spermatozoa (8). This protein has been identified as a component of the synaptonemal complex required for fulfilling meiosis and linked to mechanisms that inhibit apoptosis in male germ cells. Glucose-regulated protein 78 is closely related to HSP70-2 and shares over $61 \%$ aminoacid sequence similarity with it. In mouse testis sections, strong GRP78 staining was observed from pachytene spermatocytes to postmeiotic germ cells but not in spermatogonium and other cell types.

In the present study, we have identified that the expression of GRP78 protein is significantly higher in adult rat testes than in juvenile ones by both Western blot and immunohistochemical analysis. The current study also showed that GRP78 expression in testis increased gradually with age and its localization in different regions of the epididymis indicated the dynamic secretory mode of GRP78. The result strongly implied that GRP78 was involved in the process of mammalian spermatogenesis.

Glucose-regulated protein 78, also known as BiP, has been shown to be a molecular chaperone and $\mathrm{Ca}^{2}$ binding protein (17). It is expressed in many cell types and localized in the endoplasmic reticulum (ER). Strikingly, the transcription of GRP78 is highly induced in response to cellular stress. Potent inducers of GRP78 transcription include glucose starvation, oxygen deprivation, and treatment with thapsigargin (Tg), which depletes the $\mathrm{ER} \mathrm{Ca}^{2+}$ store. Therefore, in addition to its housekeeping functions such as protein folding, GRP78 can also serve a protective role under physiological conditions. A large number of studies have demonstrated a correlation between induced expression of GRP78 and resistance to apoptotic death in somatic cells, particularly in progressively growing tumours (13). 
Similar to tumorigenesis, spermatogenesis is a dynamic and complex process involved in massive cell proliferation and differentiation. Normal spermatogenesis is highly dependent on well-balanced germ cell proliferation, differentiation and apoptosis (18). The control of apoptosis is especially important. It plays a critical role in limiting the testicular germ cell population during male development and also serves to eliminate germ cells with altered DNA. In the adult testis, physiological apoptosis occurs at various phases of spermatogenesis. The most common pathway during germ cell death is the Fas-Fas ligand pathway and the pathway of apoptosis through the release of cytochrome c from the mitochondria, and subsequent activation of caspases have also been found essential for spermatogenesis (19). However, the molecular mechanisms that govern germ cell apoptosis are largely unknown.

Various genes have recently been demonstrated to have pro- or anti-apoptotic activities in regulating the process, including those encoding p53, transcriptional activator cyclic AMP response element modulator (CREM), HSP70-2 and several other proteins (20-22). The differential expression of GRP78 during spermatogenesis has been found in the present study, combined with its anti-apoptotic activity in other tissues, leading to the hypothesis that it may exert the same function in testis. There is accumulating evidence that the cellular distribution analysis of GRP78 in murine and human testis shows a high expression in pachytene spermatocytes. The nuclei of pachytene spermatocytes enlarge greatly as the chromosomes become shorter and thicken. Pachytene cells also exhibit an increase in RNA and protein synthesis in preparation for the next phase. These observations suggest that GRP78 performs an important function in the process of spermatogenesis.

\section{REFERENCES}

1. Mayer MP, Bukau B. Hsp70 chaperones: cellular functions and molecular mechanism. Cell Mol Life Sci 2005; 62: 670-84.

2. Lindquist S, Craig EA. The heat-shock proteins. Annu Rev Genet 1988; 22: 631-77.

3. Beere HM. "The stress of dying": the role of heat shock proteins in the regulation of apoptosis. J Cell Sci 2004; 117: 2641-51.

4. Calderwood SK, Khaleque MA, Sawyer DB. Heat shock proteins in cancer: chaperones of tumorigenesis. Trends Biochem Sci 2006; 31: 164-72.

5. Bukau B, Weissman J, Horwich A. Molecular chaperones and protein quality control. Cell 2006; 125: 443-51.
6. Nylandsted J, Brand K, Jaattela M. Heat shock protein 70 is required for the survival of cancer cells. Ann NY Acad Sci 2000; 926: 122-5.

7. Gurbuxani S, Schmitt E, Cande C. Heat shock protein 70 binding inhibits the nuclear import of apoptosis inducing factor. Oncogene 2003; 22: 6669-78.

8. Dix DJ, Allen JW, Collins BW. Targeted gene disruption of Hsp70-2 results in failed meiosis, germ cell apoptosis, and male infertility. Proc Natl Acad Sci USA 1996; 93: 3264-8.

9. Matwee C, Kamaruddin M, Betts DH. The effects of antibodies to heat shock protein 70 in fertilization and embryo development. Mol Hum Reprod 2001; 7: 829-37.

10. Kamaruddin M, Kroetsch T, Basrur PK. Immunolocalization of heat shock protein 70 in bovine spermatozoa. Andrologia 2004; 36: 327-34.

11. Spinaci $\mathrm{M}$, Volpe $\mathrm{S}$, Bernardini $\mathrm{C}$. Immunolocalization of heat shock protein $70(\mathrm{Hsp} 70)$ in boar spermatozoa and its role during fertilization. Mol Reprod Dev 2005; 72: 534-41.

12. Luo S, Mao C, Lee B. GRP78/BiP is required for cell proliferation and protecting the inner cell mass from apoptosis during early mouse embryonic development. Mol Cell Biol 2006; 26: 5688-97.

13. Reddy RK, Mao C, Baumeister P. Endoplasmic reticulum chaperone protein GRP78 protects cells from apoptosis induced by topoisomerase inhibitors: role of ATP binding site in suppression of caspase-7 activation. J Biol Chem 2003; 278: 20915-24.

14. Boilard M, Reyes-Moreno C, Lachance C. Localization of the chaperone proteins GRP78 and HSP60 on the luminal surface of bovine oviduct epithelial cells and their association with spermatozoa. Biol Reprod 2004; 71: 1879-89.

15. Lachance C, Bailey JL, Leclerc P. Expression of Hsp60 and Grp78 in the human endometrium and oviduct, and their effect on sperm functions. Hum Reprod 2007; 22: 2606-14.

16. Georgopoulos C, Welch WJ. Role of the major heat shock proteins as molecular chaperones. Annu Rev Cell Biol 1993; 9: 601-34.

17. Munro S, Pelham HR. An Hsp70-like protein in the ER: identity with the $78 \mathrm{kd}$ glucose-regulated protein and immunoglobulin heavy chain binding protein. Cell 1986; 46: 291-300.

18. Yan W, Kero J, Suominen J. Differential expression and regulation of the retinoblastoma family of proteins during testicular development and spermatogenesis: roles in the control of germ cell proliferation, differentiation and apoptosis. J Oncogene 2001; 20: 1343-56.

19. Honarpour N, Du C, Richardson JA. Adult Apaf-1-deficient mice exhibit male infertility. Dev Biol 2000; 218: 248-58.

20. Wang $\mathrm{L}$, Yeung $\mathrm{JH}, \mathrm{Hu} \mathrm{T}$, Lee WY, Lu L, Zhang $\mathrm{L}$ et al. Dihydrotanshinone induces p53-independent but ROS-dependent apoptosis in colon cancer cells. Life Sci 2013; 93: 344-51. doi: 10.1016/j.lfs.2013.07.007. Epub 2013 Jul 19.

21. Wu X, Jin W, Liu X, Fu H, Gong P, Xu J et al. Cyclic AMP response element modulator-1 (CREM-1) involves in neuronal apoptosis after traumatic brain injury. J Mol Neurosci 2012; 47: 357-67. doi: 10.1007/s12031-012-9761-1. Epub 2012 May 9.

22. Hatfield MP, Lovas S. Role of Hsp70 in cancer growth and survival. Protein Pept Lett 2012; 19: 616-24.

Submitted 18 Sep 2013

Accepted 24 Feb 2014

Published 07 Apr 2014

Online: http://myspot.mona.uwi.edu/wimjopen/article/59

(C) Wang et al 2014.

This is an open access article made freely available under Creative Commons Attribution 4.0 International (CC BY 4.0). Users are free to share, copy and adapt this work as long as the copyright holder (author) is appropriately and correctly credited. See http://creativecommons.org/ licences/by/4.0/deed.en_us for more information. 